\section{Speciation A new role for reinforcement}

\section{Smadja and R Butlin}

Heredity (2006) 96, 422-423. doi:10.1038/sj.hdy.6800826; published online 19 April 2006

$\mathrm{R}$ einforcement is a controversial mechanism of speciation in which reproductive isolation is strengthened in response to maladaptive hybridisation. In a recent paper published in Nature, Hoskin et al (2005) not only provide new empirical support for the process, in the Australian green-eyed tree frog, but also argue for a wider impact of reinforcement than is generally acknowledged.

Speciation commonly occurs in allopatry, as a by-product of natural selection and genetic drift within geographically separated populations (Figure 1a). However, an alternative hypothesis suggests that speciation may be adaptive: natural selection can act directly to favour prezygotic reproductive isolation between divergent taxa in a process known as reinforcement (Dobzhansky, 1937). If hybridisation occurs between populations that have previously diverged in allopatry, and hybrid offspring have reduced fitness, selection will favour traits that reduce the risk of hybridisation (Figure 1b). Reinforcement is theoretically plausible, and there is growing evidence for its occurrence in nature, but its role in speciation remains uncertain (Servedio and Noor, 2003). Current questions concern the factors that promote reinforcement, how frequently reinforcement plays a part in speciation and whether that part is primarily to complete speciation when isolation is already substantial (Marshall et al, 2002; Servedio, 2004).

Green-eyed tree frogs, Litoria genimaculata, form a hybrid-zone in northern Queensland. Here, Hoskin and coworkers have tested for reinforcement through a combination of molecular studies, experimental crosses, field measurements and mate choice experiments. The southern (S) and northern (N) lineages diverged genetically during a long period in allopatry and reestablished contact $\sim 5000$ years ago. They now interact in a mosaic hybrid zone that consists of a main zone of contact (A) and a second contact (B) involving a recent geographical isolate of the $S$ lineage (iS) within the range of the $\mathrm{N}$ lineage. There is strong evidence for reinforcement at contact $\mathrm{B}$, but not at the main contact A. Few studies make as complete a case for reinforcement as this one. First, Hoskin's team met Howard's (1993) demanding criteria by showing that (1) hybridisation occurs between these two lineages, (2) there is selection against hybridisation, here age, (3) male mating calls have diverged in the isolated is population (a pattern of reproductive character displacement), and (4) this divergence in mating calls is accompanied by preference of is females for males of their own lineage, leading to assortative mating between the lineages at contact B. Second, they were able to exclude one important alternative explanation for reproductive character displacement by showing that divergence in mating calls was not driven by ecological requirements (cf. Noor, 1999).

An important feature of this example is that reinforcement is restricted to the isolate (B) and is absent at the main contact, (A). The authors suggest that this is due to exposure of virtually all individuals at contact $\mathrm{B}$ to risk of hybridisation, resulting in strong selection. At the same time, the isolation of the is population from the main range of the $S$ lineage protects it from swamping by gene flow from allopatric $S$ populations. Postzygotic isolation is apparently strong enough for gene flow from $\mathrm{N}$ to be largely prevented during the early stages of reinforcement. This fits nicely with the theoretical prediction that reinforcement is most likely where there is strong selection and not too much gene flow (Servedio and Kirkpatrick, 1997); a pattern also revealed in Timema walking sticks (Nosil et al, 2003).

A novel and exciting suggestion is that natural selection acting directly against hybridisation in a contact zone can also indirectly drive rapid allopatric speciation. The authors argue that the change in frog call and mate choice in the isolated is population (due to reinforcement) has been so great that it has incidentally driven allopatric divergence between is and the genetically similar main range of the $S$ lineage. They claim that this represents the mainly affecting females of the $S$ line- rapid formation of a distinct new species of frog within the hybridzone' and a new role for reinforcement in the origin of species (Figure 1c).

Speciation requires complete reproductive isolation between the two allopatric populations of the $S$ lineage and the observation was that all $S$ and iS females chose the male call of their own group when given a choice between is and S male calls. However, this result is based on a small sample size and is surprising because the song difference between $S$ and iS males is weaker than that between iS and $\mathrm{N}$ males, where isolation by female preference is incomplete and asymmetrical. Furthermore, there is no reason to believe that the iS and $S$ populations have diverged for any traits other than call and preference. If brought back into contact by future environmental change, the lack of intrinsic postzygotic isolation and ecological differentiation suggest that they would either merge into a single population or one would out-compete the other. It is therefore questionable whether speciation is complete between iS and $S$ and it may be premature to conclude that reinforcement can be the 'sole cause of speciation' between allopatric populations.

Despite these reservations, the fact that reinforcement can drive rapid divergence between allopatric populations is an important extension of our view of the mechanisms of speciation in allopatry and the role of reinforcement. Allopatric speciation is often considered to require a long period of geographic isolation between two lineages to generate strong reproductive isolation (Figure 1a), whereas this study suggests that divergence may arise rapidly (over 5000 years) (Figure 1c). Moreover, reinforcement is usually thought to operate in completing speciation between sympatric populations after an initial period of divergence in allopatry (Figure 1b), but this example suggests a new role for reinforcement in driving prezygotic isolation between populations that remain allopatric and that have not diverged genetically (Figure 1c).

This rapid, allopatric speciation by reinforcement is related to speciation by sexual selection more generally (Panhuis et al, 2001). Selection acting directly on traits that are likely to contribute to barriers to gene flow is the key to rapid speciation. Here, reinforcement due to sympatry with the $\mathrm{N}$ lineage drives evolution in the is population and this represents a kind of sexual selection where females choose complementary mates. 


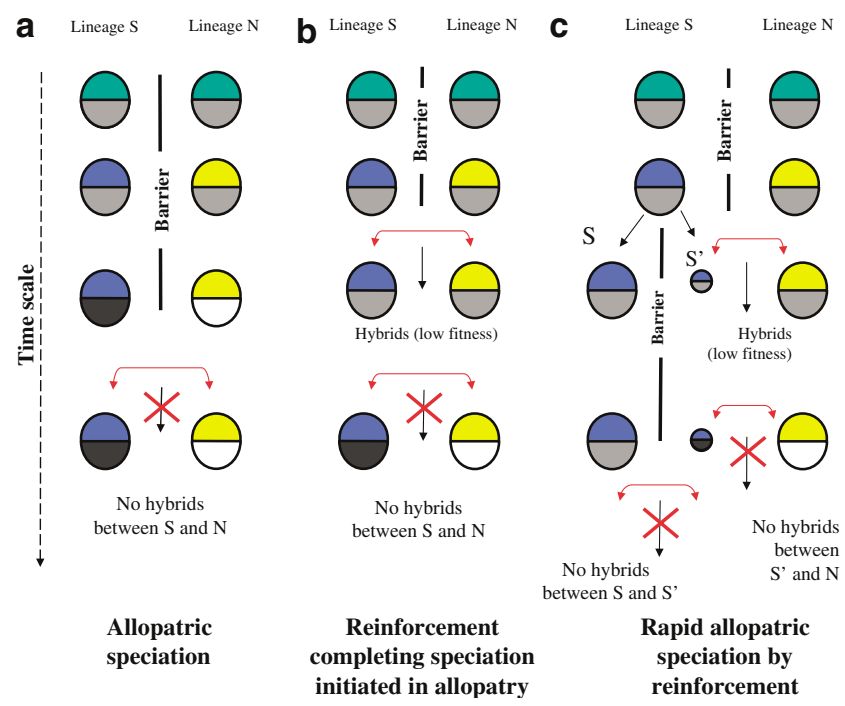

Figure 1 Three different modes of speciation between two lineages $S$ and N. In the classical view of allopatric speciation, pre- and post-zygotic isolation evolve without contact (a) whereas secondary contact promotes prezygotic isolation in the standard view of speciation by reinforcement (b). The new model (c) of rapid allopatric speciation by reinforcement, proposed by Hoskin et al (2005), suggests that reinforcement can drive speciation within lineage $\mathrm{S}$, as well as between $\mathrm{S}$ and $\mathrm{N}$.

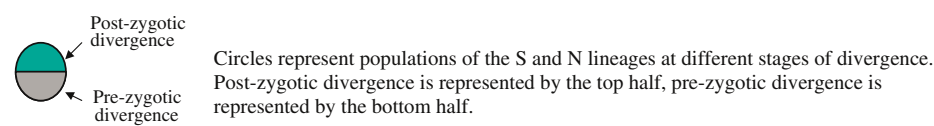

Colours represent the extent of pre-and post-zygotic divergence.

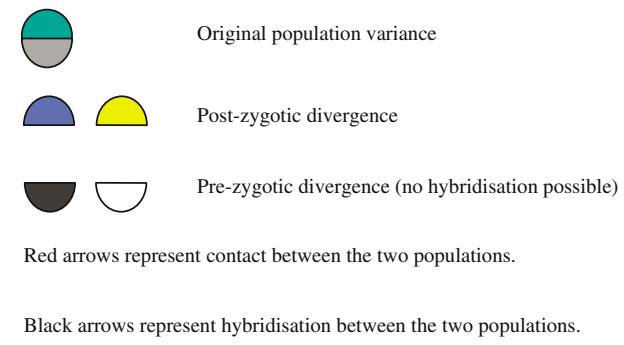

An important question for the future is whether 'rapid allopatric speciation by reinforcement' is a widespread mechanism. The green-eyed tree frog is the first example of this phenomenon in nature and only further studies will tell us if it occurs in many other species. Even if it is common that reinforcement indirectly drives divergence between allopatric isolates, it seems unlikely that this contributes much to diversity because small divergent populations rarely have long-term futures. Nevertheless, this new study suggests that reinforcement may play a wider role in speciation than was previously thought and underlines how strong regimes of selection acting on prezygotic traits, either reinforcement or sexual selection more generally, can drive rapid speciation. $R$ Butlin is at the Department of Animal and Plant Sciences, University of Sheffield, Sheffield S10 $2 T N$, UK. C Smadja is at the Laboratory of Ecology, Systematics and Evolution, University of Paris-Sud, 91405 Orsay cedex, France.

e-mail:r.k.butlin@sheffield.ac.uk

Dobzhansky T (1937). Genetics and the Origin of Species. Columbia University Press: New York.

Hoskin CJ, Higgie M, McDonald KR, Moritz C (2005). Nature 437: 1353-1356.

Howard DJ (1993) In: Harrison RG (ed) Hybrid zones and the Evolutionary Process. Oxford University Press: New York, pp 46-69.

Marshall JL, Arnold ML, Howard DJ (2002). Trends Ecol Evol 17: 558-563.

Noor MA (1999). Heredity 83: 503-508.

Nosil P, Crespi BJ, Sandoval CP (2003). Proc R Soc London Ser B-Biological Sciences 270 1911-1918.

Panhuis TM, Butlin R, Zuk M, Tregenza T (2001). Trends Ecol Evol 16: 364-371.

Servedio MR (2004). PLoS Biology 2: e420.

Servedio MR, Kirkpatrick M (1997). Evolution 51: 1764-1772.

Servedio MR, Noor MA (2003). Annu Rev Ecol Systematics 34: 339-364.

\section{Further Reading}

Mallet J (2005). Speciation in the 21st century. Heredity 95: 105-109, Book Review.

Silvertown J, Servaes C, Biss P, Macleod D (2005). Reinforcement of reproductive isolation between adjacent populations in the Park Grass Experiment. Heredity 95: 198-205, Original Article.

Jiggins CD (2006). Speciation: Reinforced butterfly speciation. Heredity 96: 107-108, News and Commentary.

Helbig AJ (2005). Evolutionary genetics: A ring of species. Heredity 95: 113-114, News and Commentary.

Ollerton J (2005). Speciation: Flowering time and the Wallace Effect. Heredity 95: 181-182, News and Commentary.

Miller NJ, Kift NB, Tatchell GM (2005). Hostassociated populations in the lettuce root aphid, Pemphigus bursarius (L.). Heredity 94 556-564, Original Article. 